\title{
A Double-Layered Covered Biliary Metal Stent for the Management of Unresectable Malignant Biliary Obstruction: A Multicenter Feasibility Study
}

\author{
Jin-Seok Park ${ }^{1}$, Seok Jeong ${ }^{1,2}$, Don Haeng Lee ${ }^{1,2,3}$, Jong Ho Moon ${ }^{4}$, Kyu Taek Lee ${ }^{5}$, and Seok Ho Dong ${ }^{6}$ \\ ${ }^{1}$ Digestive Disease Center, Department of Internal Medicine, Inha University School of Medicine, ${ }^{2}$ The National Center of Efficacy Evaluation \\ for the Development of Health Products Targeting Digestive Disorders (NCEED), ${ }^{3}$ Utah-Inha DDS \& Advanced Therapeutics Research Center, \\ Incheon, ${ }^{4}$ Digestive Disease Center and Research Institute, Department of Internal Medicine, Soonchunhyang University School of Medicine, \\ Bucheon, ${ }^{5}$ Division of Gastroenterology, Department of Medicine, Samsung Medical Center, Sungkyunkwan University School of Medicine, \\ Seoul, and ${ }^{6}$ Division of Gastroenterology and Hepatology, Department of Internal Medicine, Kyung Hee University School of Medicine, Seoul, \\ Korea
}

Background/Aims: The covered self-expandable metal stent (CMS) was developed to prevent tumor ingrowth-induced stent occlusion during the treatment of malignant biliary obstruction. However, complications such as cholecystitis, pancreatitis, and stent migration can occur after the endoscopic insertion of CMSs. The aim of the present study was to assess the efficacy and safety of a double-layered CMS (DCMS) for the management of malignant bile duct obstruction. Methods: DCMSs were endoscopically introduced into 59 patients with unresectable malignant extrahepatic biliary obstruction at four tertiary referral centers, and the patient medical records were retrospectively reviewed. Results: Both the technical and functional success rates were $100 \%$. Procedure-related complications including pancreatitis, cholangitis, stent migration, and liver abscess occurred in five patients (8.5\%). The median follow-up period was 265 days (range, 31 to 752 days). Cumulative stent patency rates were $68.2 \%$ and $40.8 \%$ at 6 and 12 months, respectively. At the final follow-up, the rate of stent occlusion was 33.9\% (20/59), and the median stent patency period was 276 days (range, 2 to 706 days). Conclusions: The clinical outcomes of DCMSs were comparable to the outcomes previously reported for CMSs with respect to stent patency period and complication rates. (Gut Liver 2016;10:969-974)

Key Words: Stents; Extrahepatic cholestasis; Feasibility studies; Common bile duct neoplasms; Adverse effects

\section{INTRODUCTION}

Endoscopic biliary stenting is a well-established method that is considered the gold standard for the palliative treatment for inoperable malignant biliary obstruction. ${ }^{1,2}$ The therapeutic effects of stent insertion are similar to those of surgical biliary drainage, but stent insertion is associated with a lower complication rate than the surgical procedure. ${ }^{3}$ Since 1979, plastic stents have been widely used to allow biliary drainage in cases of malignant biliary obstruction. ${ }^{3-5}$ However, stent failure due to clogging caused by protein in bile juice, bacterial growth, or biliary secretion frequently occurs after a short period of time. Therefore, stent replacement every 3 to 4 months is required to maintain adequate biliary drainage. ${ }^{5}$ Self-expandable metal stents (SEMSs) are more expensive than plastic stents but remain patent for longer because of their larger diameters, which can be extended to 24-36 F. This results in a lower incidence of bacterial growth in the stent. However, SEMS failure usually occurs because of tissue hyperplasia or neoplastic tumor ingrowth through the stent mesh, ${ }^{6}$ and thus, covered self-expanding metal stent (CMS) with a membrane that prevents tissue ingrowth into the stent, were developed to overcome the drawbacks of SEMS. Nevertheless, although CMSs may increase stent patency related to tissue ingrowth, stent occlusion associated with bile sludge clogging or tumor overgrowth can occur. ${ }^{7,8}$ In addition, CMSs can cause complications associated with the stent-covering such as pancreatitis, cholecystitis, and stent migration. ${ }^{8,9}$ CMSs are also more likely to migrate due to a lack of tissue embedding. ${ }^{10,11}$

\footnotetext{
Correspondence to: Seok Ho Dong

Division of Gastroenterology and Hepatology, Department of Internal Medicine, Kyung Hee University School of Medicine, 26 Kyungheedae-ro, Dongdaemun-gu, Seoul 02447, Korea

Tel: +82-2-958-8200, Fax: +82-2-968-1848, E-mail: gidrdong@hanmail.net

Received on March 4, 2015. Revised on September 15, 2015. Accepted on December 14, 2015. Published online May 16, 2016 pISSN 1976-2283 eISSN 2005-1212 https://doi.org/10.5009/gnl15112

Jin-Seok Park and Seok Jeong contributed equally to this work as first authors.

@) This is an Open Access article distributed under the terms of the Creative Commons Attribution Non-Commercial License (http://creativecommons.org/licenses/by-nc/4.0) which permits unrestricted non-commercial use, distribution, and reproduction in any medium, provided the original work is properly cited.
} 

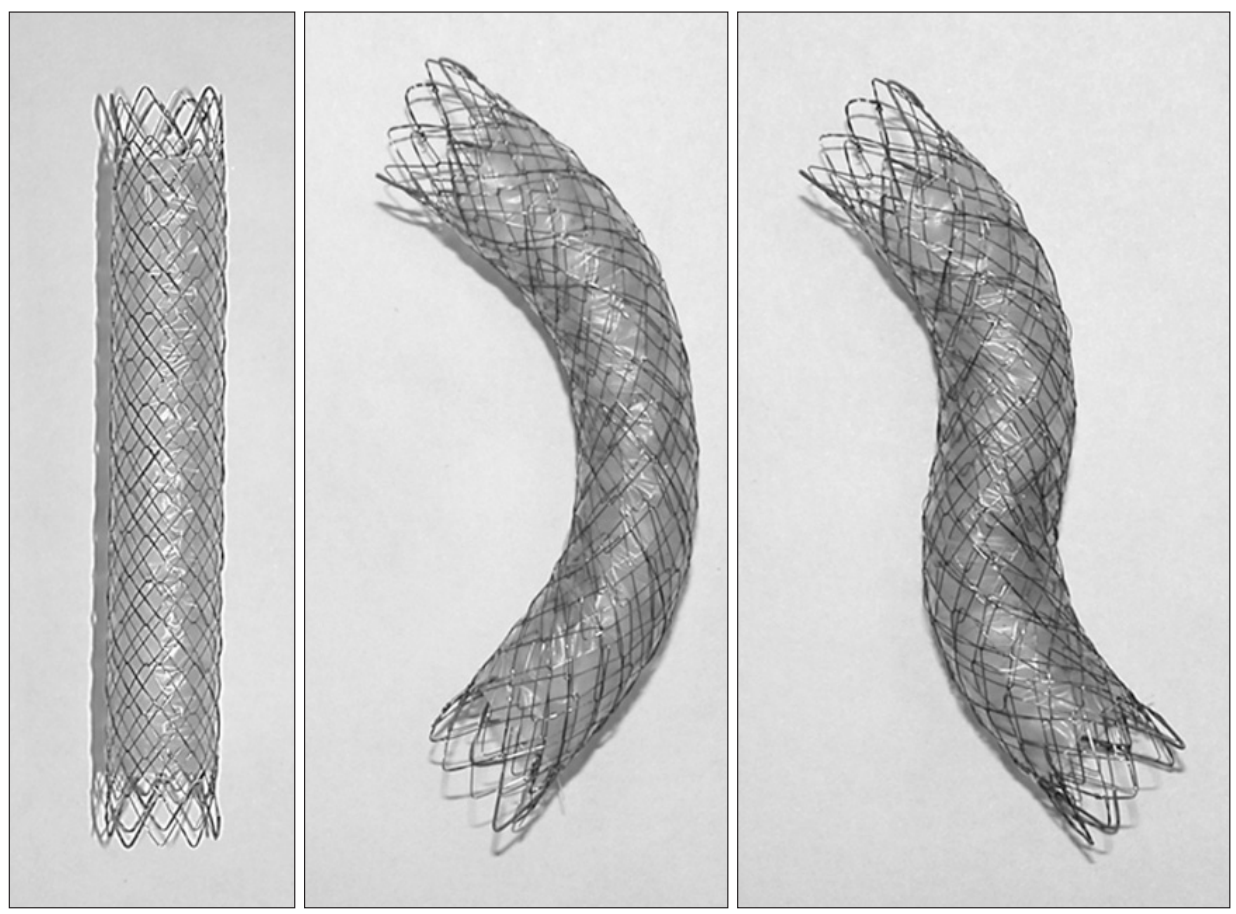

Fig. 1. The double-layered, covered metal stent $\left(\mathrm{EGIS}^{\mathrm{TM}}\right.$; S\&G BioTech Inc.) consists of a silicone membrane sandwiched between two nitinol self-expanding metallic stent layers. The cell size of this stent is smaller than that of other covered metal stents, because the use of the nitinol suture allows the two attached stent layers to partially overlap.

The biliary double-layered, covered metal stent (DCMS) was designed to reduce tissue ingrowth and stent covering-related complications. The purpose of this retrospective study was to evaluate the efficacy and safety of the DCMS for the palliative treatment of malignant extrahepatic biliary obstruction.

\section{MATERIALS AND METHODS}

\section{Patients}

DCMSs were endoscopically placed to treat unresectable malignant common bile duct obstruction at four tertiary referral centers between October 2007 and November 2011. All patients treated were enrolled in the present study and followed up, and clinical courses were recorded at each center. The study protocol was approved by the Institutional Review Board of all participating centers. The inclusion criteria were (1) age $>18$ years, (2) inoperable malignant extrahepatic biliary obstruction, (3) no previous attempt of endoscopic biliary drainage, and (4) no history of biliary tract surgery. The exclusion criteria were (1) intrahepatic biliary obstruction and (2) inadequate medical records. The medical records of the patients were analyzed and the following information was extracted: clinical characteristics, stent dysfunction, stent-related complications, and clinical outcome.

\section{Stent and procedure}

The structure of the DCMS (S\&G BioTech Inc., Seongnam, Korea) used is shown in Fig. 1. The stent consists of a silicone membrane sandwiched between two nitinol stents. Since the cell of a nitinol stent partially overlaps with the cell of another

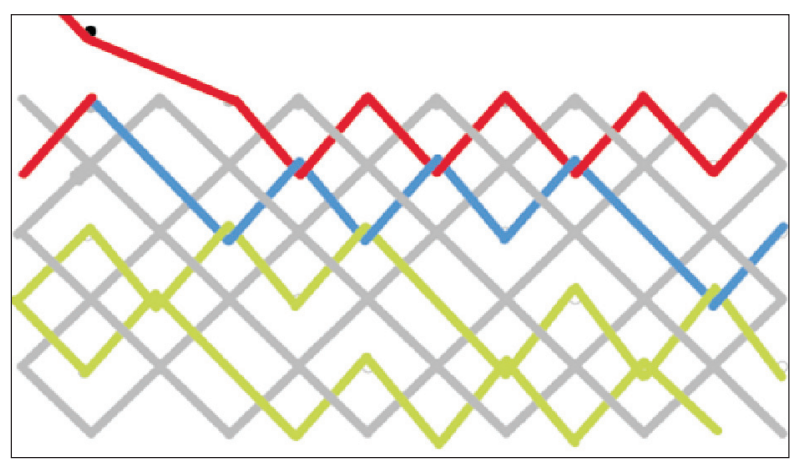

Fig. 2. V-type wire. The colored lines denote the V-shaped wire, and the gray lines denote the basic mesh structure of the stent. The incorporation of the V-shaped wire, which has a zigzag shape, reduces the axial force and shortening of the stent.

nitinol stent, each cell size of DCMS is small compared to that of other CMSs. This is expected to reduce the risk of tissue hyperplasia and tumor ingrowth. Furthermore, the DCMS contains a special structure to reduce axial forces of the stent, which can straighten stents or result in the proximal end of a stent being pushed against the bile duct wall during stent insertion. A specially weaved V-shaped wire provides the stent with a flexible structure. A usual nitinol stent consists of two wires that are twisted together. Its mesh construction tends to be easily shortened, and it has a strong axial force. Adding the V-shaped wire to the twisted structure may add to its ability to resist axial forces and increase the radial force, and thus, prevent stent shortening (Fig. 2).

All stents were inserted into bile ducts by experienced pan- 
creaticobiliary endoscopists using a routine procedure during endoscopic retrograde cholangiopancreatography. Endoscopic sphincterotomy was performed before stent insertion. Technical and functional success rates and complications associated with stent placement were evaluated. Technical success was defined as the successful placement of a stent across a stricture. Functional success was defined as an improvement in bilirubin to less than $75 \%$ of the pretreatment value within a month of the procedure. Early and late complications were defined as those occurring at $<30$ days or $>30$ days after stent placement, respectively.

\section{Follow-up and end points}

Patients visited hospital monthly to three months after stent placement to assess stent function, and were examined for symptoms and signs of biliary obstruction. Blood tests were performed at each visit. Computed tomography (CT) was performed to assess stent dysfunction when biliary obstruction was suspected. The primary study end point was stent dysfunction, which was defined as the presence of symptoms of biliary obstruction, that is, abdominal pain, dark urine, fever, jaundice, nausea and vomiting, acholic stools or an increased bilirubin level with an abnormal liver profile by blood testing and confirmation by cholangiography or CT. Tumor ingrowth was defined as growth of the tumor invading the body of the stent and occluding its lumen on cholangiography. Likewise, tumor overgrowth was defined as growth of the tumor proximal or distal to stent and leading the lumen occlusion with function loss. Stent patency period was defined as the time elapsed between placement and occlusion. When a stent remained patent until

Table 1. Types of Malignancy Causing Obstruction

\begin{tabular}{lc}
\hline \multicolumn{1}{c}{ Type of malignancy } & No. (\%) \\
\hline Pancreatic & $35(59.3)$ \\
Primary bile duct & $12(20.3)$ \\
Gall bladder & $4(6.8)$ \\
Ampullary & $4(6.8)$ \\
Other cancers & \\
Lung & $2(3.4)$ \\
Stomach & $1(1.7)$ \\
Unknown origin & $1(1.7)$ \\
Total & 59 \\
\hline
\end{tabular}

Table 2. Location of Extrahepatic Obstructions

\begin{tabular}{lc}
\hline Location of obstruction & No. (\%) \\
\hline Distal common bile duct & $34(57.6)$ \\
Mid common bile duct & $17(28.8)$ \\
Proximal common bile duct & $8(13.6)$ \\
Total & 59 \\
\hline
\end{tabular}

death, the patency period was measured from date of placement to death. The secondary end point was stent migration rate. Migration was defined as movement of the stent to a position where it was no longer satisfactorily bridging the stricture. Stent migration period was measured from the date of stent insertion to the date of stent migration detection. Patient survival was defined as time from diagnosis to death. When a patient was lost to follow-up or date of death was unascertained, survival period was calculated from diagnosis to date of last visit. Cumulative patient survival and stent patency were calculated using the Kaplan-Meier method and SPSS version 19.0 (IBM Corp., Armonk, NY, USA). The stent patency and survival periods of patients who were lost to follow-up or died during the study period were censored.

\section{RESULTS}

Fifty-nine patients with malignant extrahepatic biliary stricture that underwent endoscopic biliary drainage with the DCMS between October 2007 and November 2011 were retrospectively enrolled in this study. The etiologies of malignancies are summarized in Table 1. In all patients, malignancy was confirmed by histopathologic examination. Median age of the patients was 70 years (range, 43 to 87 years), and the male:female ratio was approximately 3:2 (36:23 cases). Median follow-up was 265 days (range, 31 to 752 days). Pancreatic cancer was the most common cause of malignant extrahepatic biliary stricture, and the distal CBD was most commonly affected (Table 2).

The DCMS was successfully deployed in all 59 cases, and technical and functional success rates were both 100\%. Symptoms of biliary obstruction improved in all cases. Complications occurred in five patients (8.5\%), and early complications in two of these five patients. Acute cholangitis occurred in one patient but responded to antibiotics without further complication. In one patient, stent migration was observed 2 days after

Table 3. Reasons for Stent Dysfunction and Stent-Associated Complications

\begin{tabular}{ll}
\hline & No. (\%) \\
\hline Stent dysfunction & $20(33.9)$ \\
Ingrowth & 4 \\
Overgrowth & 3 \\
Sludge & 13 \\
Complications & $5(8.5)$ \\
Early complications & \\
Cholangitis & 1 \\
Migration & 1 \\
Delayed complications & \\
Liver abscess & 1 \\
Migration & 2 \\
\hline
\end{tabular}


placement, but after repositioning related symptoms and signs resolved. Asymptomatic hyperamylasemia occurred in two patients. Late complications occurred in three; stent migration in two patients and a liver abscess in one. These two late complications were cause by distal and proximal stent migration at 162 and 156 days, respectively, after placement. The distal migration resolved after replacement with a new DCMS, and the proximal migration was successfully treated by additional metal stent insertion without previous stent removal. The liver abscess was treated with antibiotics without additional biliary drainage (Table 3). Consequently, stent migration occurred in a total of three patients and all of them could be treated by stent repositioning or additional stent insertion. In this study, cholecystitis did not occur in any patients.

Median stent patency was 276 days (range, 2 to 706 days), and stent dysfunction was observed in $33.9 \%$ of cases (20/59). Cumulative stent patency rates were $68.2 \%$ at 6 months and $40.8 \%$ at 12 months after placement (Fig. 3). Causes of stent dysfunction were bile sludge (13/20, 65\%), tumor ingrowth (4/20, 20\%), and tumor overgrowth (3/20, 15\%). Bile sludge was removed by mechanical cleaning with an extraction balloon catheter in seven patients, and in the other six patients additional biliary drainage was required to correct the stent dysfunction (a metal stent was used in four, a plastic stent in one, and percutaneous transhepatic biliary drainage in one). Among 13 patients, stent clogging by biliary sludge occurred 2

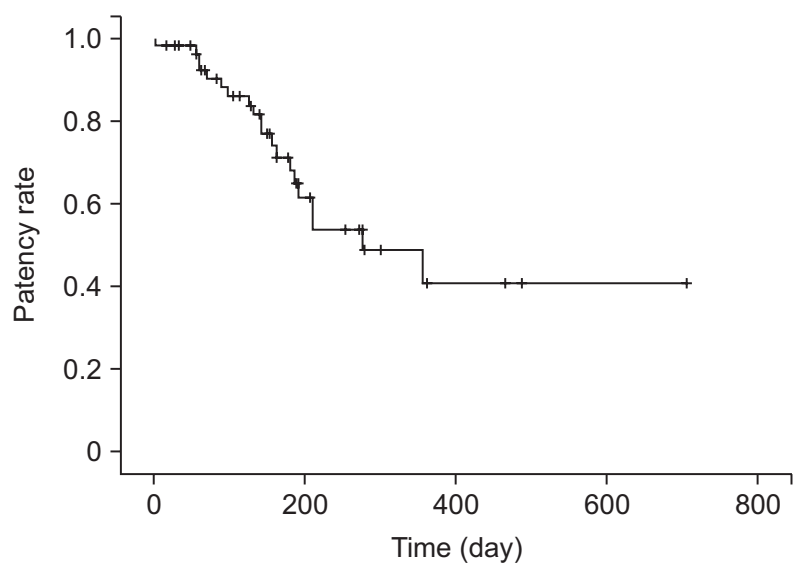

Fig. 3. Kaplan-Meier analysis of double-layered, covered metal stent patency rates. The median stent patency period was 276 days, and the cumulative stent patency rates were $68.2 \%$ at 6 months and $40.8 \%$ at 1 year. days after stent insertion in one patient and the stent also could recover the patency by mechanical cleansing. Tumor ingrowth and overgrowth required additional metal or plastic stent insertions. For the 59 patients, overall stent dysfunction occurred at median periods of 158 days placement (Table 4). An analysis of time to stent dysfunction by cause showed that bile sludge (median days, 126) tended to occur faster than tissue ingrowth (189 days) or overgrowth (142 days).

Of the 59 patients, 56 were followed-up until death; median survival was 253 days (range, 15 to 752 days). Patient survival dropped rapidly during the early phase of follow-up (6-month cumulative survival rate, 58.9\%), but then slowed (1-year cumulative survival rate, 26\%) (Fig. 4). However, stent related death did not occur in this study. All stent dysfunctions were treated with endoscopic cleansing or additional biliary drainages.

\section{DISCUSSION}

The present study shows that DCMS placement is an effective palliative treatment for malignant biliary stricture; technical and functional success rates were both 100\%.

The overall complication rate related to DCMS placement was $8.5 \%$, which is similar to those reported by other studies on CMSs (Table 5). Pancreatitis has been reported to be more common in patients that have received a CMS, ${ }^{8}$ but the rate of pancreatitis was low in the present study. Furthermore, the

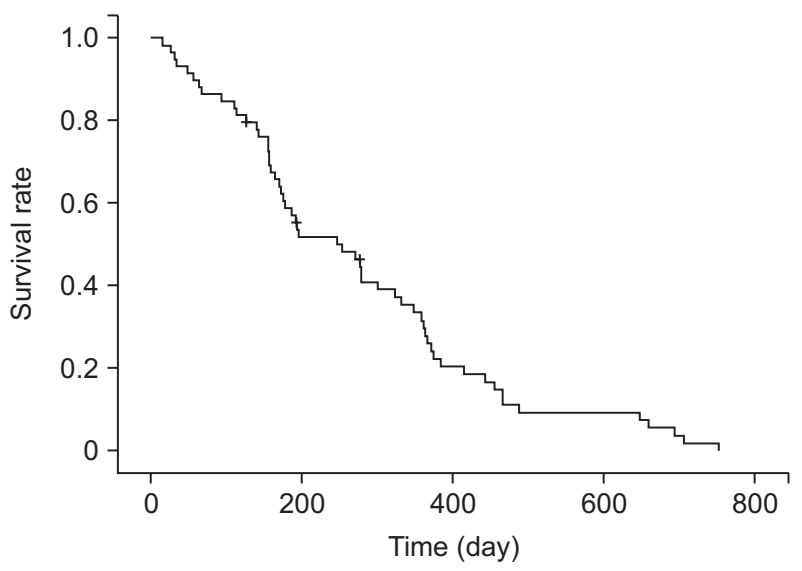

Fig. 4. Kaplan-Meier analysis of survival rates. The mean survival period was 253 days, and the cumulative survival rates were $58.9 \%$ at 6 months and 26\% at 1 year.

Table 4. Stent Patency according to the Cause of Stent Obstruction

\begin{tabular}{lccc}
\hline \multicolumn{1}{c}{ Reason } & No. & Mean stent patency (SD), day & Median stent patency (range), day \\
\hline Tumor ingrowth & 4 & $208.5(45.3)$ & $189(180-276)$ \\
Tumor overgrowth & 3 & $201.0(111.9)$ & $142(131-330)$ \\
Sludge & 13 & $133.5(90.7)$ & $126(2-210)$ \\
Total & 20 & $158.6(89.9)$ & 149 \\
\hline
\end{tabular}


Table 5. Results of Trials Evaluating CMSs for the Treatment of Malignant Biliary Obstruction

\begin{tabular}{|c|c|c|c|c|c|c|}
\hline Study & $\begin{array}{c}\text { No. of } \\
\text { patients }\end{array}$ & Type of stent & Survival, day & Patency, day & $\begin{array}{c}\text { Total no. of } \\
\text { complications (\%) }\end{array}$ & Follow-up \\
\hline Kahaleh et al. ${ }^{18}$ & 80 & Covered Wallstent & $177^{\dagger}$ & $171^{\dagger}$ & $7(8.7)$ & $3 \mathrm{mo}^{*}$ \\
\hline Nakai et al. ${ }^{11}$ & 69 & Covered Wallstent & $200^{\dagger}$ & $139^{\dagger}$ & $9(13)$ & $2 \mathrm{yr}^{+}$ \\
\hline Isayama et al. ${ }^{8}$ & 47 & Covered ComVi & $255^{\dagger}$ & $208^{\dagger}$ & $2(4.2)$ & NA \\
\hline Telford et al. ${ }^{16}$ & 68 & Covered Wallstent & $227^{*}$ & $357^{*}$ & $19(27)$ & 201 day* \\
\hline Perri et al. ${ }^{19}$ & 70 & Covered ComVi & $190^{*}$ & $154^{*}$ & $5(7.1)$ & $12 \mathrm{mo}^{*}$ \\
\hline Present study & 59 & $\begin{array}{c}\text { Biliary double-layered and } \\
\text { membrane covered stent }\end{array}$ & $253^{*}$ & $276^{*}$ & $5(8.5)$ & 265 day* $^{*}$ \\
\hline
\end{tabular}

NA, not assessable.

*Values are expressed as medians; ${ }^{\dagger}$ Values are expressed as means.

incidence of stent migration was only 5\% (three patients) even though DCMS does not have fixing equipment in the CBD, such as anchoring flap and flared end. ${ }^{12}$ We believe that the lower incidence of complications observed in this study is related to the V-shaped structure of the stent, which reduces axial forces applied to DCMSs.

Accordingly, DCMSs can be placed along the course of the bile duct because this reduction in axial force would tend to prevent stent migration and the incidence of complications related to stent insertion. Isayama et al. ${ }^{13}$ analyzed the stent patency of the ComVi stent, which has lower axial forces and better resists radial compressive forces than other CMSs. In this previous study, stent migration occurred in only one patient with a ComVi stent (2.1\%), and no case of pancreatitis was encountered. Furthermore, these results are superior to those obtained for the covered Wallstent (17\%). These results suggest that in-stent axial force is an important factor of stentrelated complications and we believe that the design to reduce axial force of stent in DCMS also affect the low rate of adverse event including migration. However, few studies have analyzed the incidences of complications associated with in-stent axial force, and thus, further prospective studies are needed to confirm our results. Cholecystitis is an important complication of CMS, and occlusion of the cystic duct by a CMS is a potential risk factor of cholecystitis. According to previous reports, the incidence of cholecystitis in CMS insertion ranges between 3\% and $10 \% .{ }^{14,15}$ In our series, no patient developed acute cholecystitis after DCMS placement. However, we estimate that this result was more affected by our careful endoscopic procedures than the function of DCMS, in which all DCMSs used current study placed below the cystic duct, and not cross the cystic duct. Therefore, clinicians have to pay attention to measure the accurate stricture length and to choose an appropriate length of stent when they use the CMSs for treatment of biliary stricture.

In the present study, the rate of DCMS dysfunction among the 59 study subjects was 33.9\%, and sludge was the main cause of dysfunction (13/20). Despite the fact that the DCMS has special features to reduce stent dysfunction by sludge such as a pol- ished inner layer to prevent sludge accumulation, the incidence of stent dysfunction caused by sludge formation remained high, which represents a limitation regarding the use of DCMSs to manage malignant biliary obstruction.

Tumor ingrowth was observed in four patients (6.8\%) in the present study, which is similar or slightly lower than that found in other CMS studies. ${ }^{8,16,17}$ In fact, it has been reported that tumor ingrowth occurs in $8.8 \%$ to $16 \%$ of CMSs. ${ }^{13,15}$ The DCMS used in the present study was developed to suppress tumor ingrowth by increasing surface tension through a reduction in the cell size. The cells of DCMSs are $2 \mathrm{~mm}$ in diameter, whereas the cells of other CMSs are approximately $4 \mathrm{~mm}$ in diameter. In addition, a silicone membrane is placed between two layers of nitinol wires. In the present study, tissue ingrowth occurred in four patients after a mean period of 208 days, which could have been due to loss of silicone membrane integrity after nearly 7 months. However, the integrity of silicone membrane have not been studied in current study. Therefore, the futher study would be needed to verify the our hypothesis. In the present study, the median stent patency period was 276 days, which is similar or slightly longer than values reported in studies on CMSs (Table 5). ${ }^{8,10,11,15,18}$ However, the 6-month patency rate in the present study was $68.2 \%$, which is lower than those of previous relevant series, which reported rates ranging from 78\% to $93 \%{ }^{19}$ We believe that this result could be associated with stent dysfunction rates. Stent dysfunction occurred in 15 cases within 6 months, and subsequently, only five cases were reported during the 2-year follow-up. Stent dysfunction caused by bile sludge tended to occur earlier than those caused by other factors; in fact, most cases of stent dysfunction within the first 6 months were caused by bile sludge. Thus, longer DCMS patency would be expected if bile sludge accumulation could be inhibited in the stent lumen.

This study has several limitations that warrant consideration. First, the study is limited by its retrospective nature, and as a result, we were not able to enroll sufficient patients to precisely evaluate the efficacy of the stent. Second, the study has only a single arm, and thus, the DCMS could not be compared with 
other biliary stents.

In conclusion, the DCMS was found to have the ability to prevent tissue ingrowth and stent migration. In addition, its patency and complication rates were similar to those reported for CMSs. However, stent dysfunction caused by bile sludge occurred frequently, and this aspect of the DCMS requires further research effort to identify an effective solution.

\section{CONFLICTS OF INTEREST}

No conflict of interest relevant to this article was reported.

\section{ACKNOWLEDGEMENTS}

This work was supported by Inha University Hospital Research Grant.

Authors' contributions: J.S.P. and S.J.: conception and design, collection and assembly of data, analysis and interpretation of the data, drafting of the article, provision of study materials, administrative, technical, and logistic support. D.H.L., J.H.M., and K.T.L.: collection and assembly of data and the provision of study materials or patients. S.H.D.: conception and design, collection and assembly of data, critical revision of the article for important intellectual content, administrative aspects, and final approval of the article.

\section{REFERENCES}

1. Speer AG, Cotton PB, Russell RC, et al. Randomised trial of endoscopic versus percutaneous stent insertion in malignant obstructive jaundice. Lancet 1987;2:57-62.

2. Petersen BT, Kahaleh M, Kozarek RA, et al. A multicenter, prospective study of a new fully covered expandable metal biliary stent for the palliative treatment of malignant bile duct obstruction. Gastroenterol Res Pract 2013;2013:642428.

3. Terruzzi V, Comin U, De Grazia F, et al. Prospective randomized trial comparing Tannenbaum Teflon and standard polyethylene stents in distal malignant biliary stenosis. Gastrointest Endosc 2000;51:23-27.

4. Soehendra N, Reynders-Frederix V. Palliative biliary duct drainage: a new method for endoscopic introduction of a new drain. Dtsch Med Wochenschr 1979;104:206-207.

5. England RE, Martin DF, Morris J, et al. A prospective randomised multicentre trial comparing $10 \mathrm{Fr}$ Teflon Tannenbaum stents with 10 Fr polyethylene Cotton-Leung stents in patients with malignant common duct strictures. Gut 2000;46:395-400.

6. Jaganmohan S, Lee JH. Self-expandable metal stents in malignant biliary obstruction. Expert Rev Gastroenterol Hepatol 2012;6:105114.

7. Shim CS, Lee YH, Cho YD, et al. Preliminary results of a new covered biliary metal stent for malignant biliary obstruction. Endoscopy 1998;30:345-350.

8. Isayama H, Komatsu Y, Tsujino T, et al. A prospective randomised study of "covered" versus "uncovered" diamond stents for the management of distal malignant biliary obstruction. Gut 2004;53: 729-734.

9. Fumex F, Coumaros D, Napoleon B, et al. Similar performance but higher cholecystitis rate with covered biliary stents: results from a prospective multicenter evaluation. Endoscopy 2006;38:787-792.

10. Park do H, Kim MH, Choi JS, et al. Covered versus uncovered wallstent for malignant extrahepatic biliary obstruction: a cohort comparative analysis. Clin Gastroenterol Hepatol 2006;4:790-796.

11. Nakai Y, Isayama H, Komatsu Y, et al. Efficacy and safety of the covered Wallstent in patients with distal malignant biliary obstruction. Gastrointest Endosc 2005;62:742-748.

12. Park do H, Lee SS, Lee TH, et al. Anchoring flap versus flared end, fully covered self-expandable metal stents to prevent migration in patients with benign biliary strictures: a multicenter, prospective, comparative pilot study (with videos). Gastrointest Endosc 2011;73:64-70.

13. Isayama $\mathrm{H}$, Kawabe $\mathrm{T}$, Nakai $\mathrm{Y}$, et al. Management of distal malignant biliary obstruction with the ComVi stent, a new covered metallic stent. Surg Endosc 2010;24:131-137.

14. Yoon WJ, Lee JK, Lee KH, et al. A comparison of covered and uncovered Wallstents for the management of distal malignant biliary obstruction. Gastrointest Endosc 2006;63:996-1000.

15. Suk KT, Kim HS, Kim JW, et al. Risk factors for cholecystitis after metal stent placement in malignant biliary obstruction. Gastrointest Endosc 2006;64:522-529.

16. Telford JJ, Carr-Locke DL, Baron TH, et al. A randomized trial comparing uncovered and partially covered self-expandable metal stents in the palliation of distal malignant biliary obstruction. Gastrointest Endosc 2010;72:907-914.

17. Kullman E, Frozanpor F, Söderlund C, et al. Covered versus uncovered self-expandable nitinol stents in the palliative treatment of malignant distal biliary obstruction: results from a randomized, multicenter study. Gastrointest Endosc 2010;72:915-923.

18. Kahaleh M, Tokar J, Conaway MR, et al. Efficacy and complications of covered Wallstents in malignant distal biliary obstruction. Gastrointest Endosc 2005;61:528-533.

19. Perri V, Boškoski I, Tringali A, et al. Prospective evaluation of the partially covered nitinol "ComVi" stent for malignant non hilar biliary obstruction. Dig Liver Dis 2013;45:305-309. 